\title{
A Guide to Performing Seated, Foot-On-Floor Position Ultrasound Guided Knee Injections Targeting the Medial \& Lateral Parapatellar Bursae \& Their Channels
}

\author{
James Inklebarger ${ }^{1}$, Luna DG $^{2}$, Galanis $\mathbf{N}^{3}$, Akhtar $\mathrm{A}^{4}$, Rimbault $\mathbf{T}^{5}$, Joshi $\mathbf{T}^{6}$, Gyer $\mathbf{G}^{7}$, Michael $\mathbf{J}^{8}$, \\ Bernardotto $\mathbf{M}^{9}$ \\ Sports \& MSK Physician Ravenscroft Health Centre 221 Marsh Rd, Pinner HA5 5NE, United Kingdom \\ Info@Ravenscrofthealth.co.uk \\ E-mail Address: james.inklebarger@yahoo.co.uk
}

\begin{abstract}
:
Ultrasound guided (USG) supra patellar bursa (SPB) knee aspirations-injections are a current standard practice, typically performed with the patient supine on a plinth, and with the knee flexed to around 30 degrees. This follows research that 30 degrees of knee flexion offers optimal SPB bursal resolution. However, in circumstances of pain-mobility issues affecting plinth transfer, an alternative seated, foot on floor or wheelchair pedal, knee-flexed 90 degrees procedure (SKIF) is proposed. This foot on floor position may facilitate a push-off counter resistance, facilitating isometric quadriceps contraction enhanced suprapatellar bursa (SPB) viewing, along with improved resolution of the lesser described medial-lateral parapatellar bursae (MPPB-LPPB) and their communicating SPB recess channels. As the MPPB-LPPB interconnect to the medial-lateral SPB recesses but are located more superficially and centrifugal to the femoral condyles in areas devoid of underlying articular cartilage, their preferential SKIF injectionaspiration, may also prevent cartilage needle stick injuries. also known as the paracondylar gutters, the relatively superficial MPPB-LPPB position may likewise offer better image resolution, lessen needle travel distances and needle trajectory re-directs, which may in turn enhance procedure safety, comfort, and speed. Though the medial-lateral SPB recesses have some mention in the literature, it would appear that inter tribursal connecting channels linking the MPPB-LBBP to the SPB recesses have not previously been described. This report explores tri-bursal and inter-bursal channel anatomy, relevant to performing USG $\underline{\text { SKIF injections and also elucidates the method, potential advantages, and pitfalls of this novel technique. }}$
\end{abstract}

Key Words: Parapatellar Bursa, Paracondylar Recesses, Suprapatellar, Channels, Ultrasound, Injection

\section{Introduction}

A bursa is a fluid-filled structure that is present between the skin and tendon or tendon and bone. The main function of a bursa is to reduce friction between adjacent moving structures. [1]

The suprapatellar bursa (SPB) is a major bursa of the knee.

During fetal life, a suprapatellar septum between the knee joint cavity and SPB forms and then usually perforates, establishing communication between them. The SPB may however present with morphological variance, [2] and is referenced by other names such as the SP recess, pouch, gutter, or space. [3]

Ultrasound guided (USG) supra patellar bursa (SPB) knee aspirations-injections are a current standard practice, typically performed with the patient supine on a plinth, with the knee flexed to around 30 degrees and a transverse transducer position. This follows research that 30 degrees of knee flexion offers optimal SPB bursal resolution. However, in this study SPB, fluid was evaluated in the sagittal rather than transverse plane [4]

Other studies have noted that quadriceps isometric voluntary contraction enhances tri-bursal visibility, and surface EMG bench push-off studies have reported that quadriceps isometric contraction overall occurs maximally at 90 degrees knee flexion. [5] 
In circumstances of pain-mobility issues affecting plinth transfer, an alternative seated, foot on floor or wheelchair pedal knee-flexed 90 degrees procedure (SKIF), tri-bursal injection position may hold other advantages, such as redistribution fluid transfer and subsequent improved resolution of the medial-lateral parapatellar bursae (MPPB-LPPB) and their communicating SPB recess channels. As the MPPB-LPPB interconnect with the medial-lateral SPB recesses but are located more superficially and centrifugal to the femoral condyles in areas devoid of underlying articular cartilage, preferential SKIF injection-aspiration, may also prevent cartilage needle stick injuries. Their para condylar position may likewise offer better image resolution, lessen needle travel distances and needle trajectory re-directs, which may in turn enhance procedure safety, comfort, and speed. In some cases, the SPB and its recesses may also present as unexpanded potential spaces, or be obscured by hypertrophic synovial overgrowth or other reasons, challenging echo-delineation of the bursa from surrounding fat pads and adjacent underlying intracondylar articular cartilage. The MPPB-LPPB or their channels may therefore be a good targeting choice, in cases were SPB visualization is challenged or visa-versa. The SKIF foot on floor or wheelchair pedal position [Photos 1 \& 2] may also facilitate quadriceps isometric contraction, which enhances tri-bursal and bursal channel and US imaging. Though the medial-lateral SPB recesses are described in the literature, it would appear that their LPPB-LPPB are less known, while formal anatomical descriptions of inter tri-bursal connecting channels linking the MPPB-LPPB to the lateral recesses of the SPB appear to have gone unreported. This paper explores the tri-bursal and inter-bursal channel anatomy relevant to SKIF USG injection.

SBP, MPPB-LBBP, Recess and Communicating Channel Anatomy

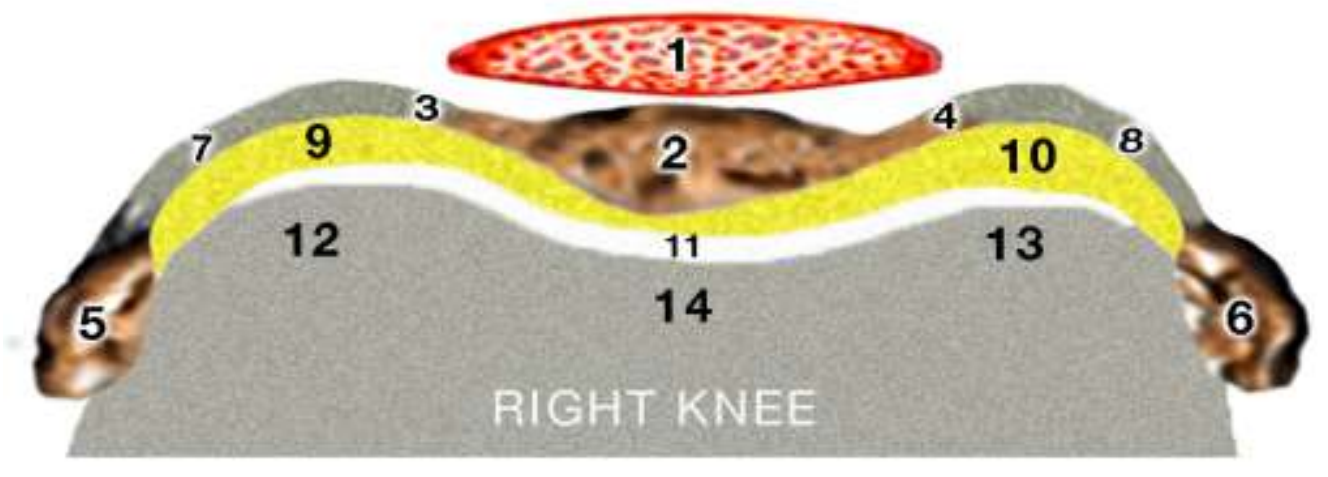

1. Quadriceps Tendon (QT)

2. Supra patellar Bursa (SPB)

3. Lateral Supra Patellar Recess (LSPR)

4. Medial Supra Patellar Recess (MSPR)

5. Lateral Parapatellar Bursa (LPPB)

6. Medial Parapatellar Bursa (MPPB)

7. Lateral Parapatellar Bursa Channel

8. Medial Parapatellar Bursa Channel

9. Lateral Supracondylar Fat Pad (LSFP)

10. Medial Supracondylar Fat Pad (MSFP)

11. Articular Cartilage

12. Lateral Femoral Condyle (LFC)

13. Medial Femoral Condyle (MFC)

14. Trochlear Notch

Figure 1: Suprapatellar Tri-Bursal Anatomy 
James Inklebarger et. al. / A Guide to Performing Seated, Foot-On- Floor Position Ultrasound Guided Knee Injections Targeting the Medial \& Lateral Para patellar Bursae \& Their Channels

Sometimes referred to as para-condylar spaces, [6. Marco A 2013] or recesses, the MPPB-LBBP are variably present structures sparsely documented in the literature. Pneumo-arthrography studies have described the LBBP as a saccular-shaped structure, occurring lateral to the suprapatellar bursa in just under $20 \%$ of the knee joints. [2]

Though no corresponding medial suprapatellar recess was identified in the Zidom \& colleagues study, an ultrasound effusion study by Mandl et al, noted and classified 3 major synovial fluid recesses as suprapatellar, medial parapatellar, and lateral parapatellar. [2, 4]

When present, the MPPB-LPPB are interconnected in a tri-bursal complex, via communicating channels to the medial \& lateral SPB recesses. These intra bursal channel structures imaged in Figure 1, Images 6, 7, 8, 10 , appear to have not previously been described in the literature. These interconnecting channels follow the contours of the medial \& lateral epicondyles, and connect to the MPPB-LPPB to the medial-lateral SP recesses. As these channels appear to have no prior anatomical literature reference, this paper will delineate the central SP recesses from the more peripheral channels using the mid-points of the medial \& lateral femoral condyles as their division. Since the very existence of the MPPB appears to of some controversy, several images capturing both the MPPB-LPPB and channel anatomy have been presented in Images 1, 6, 7, 8,10 .

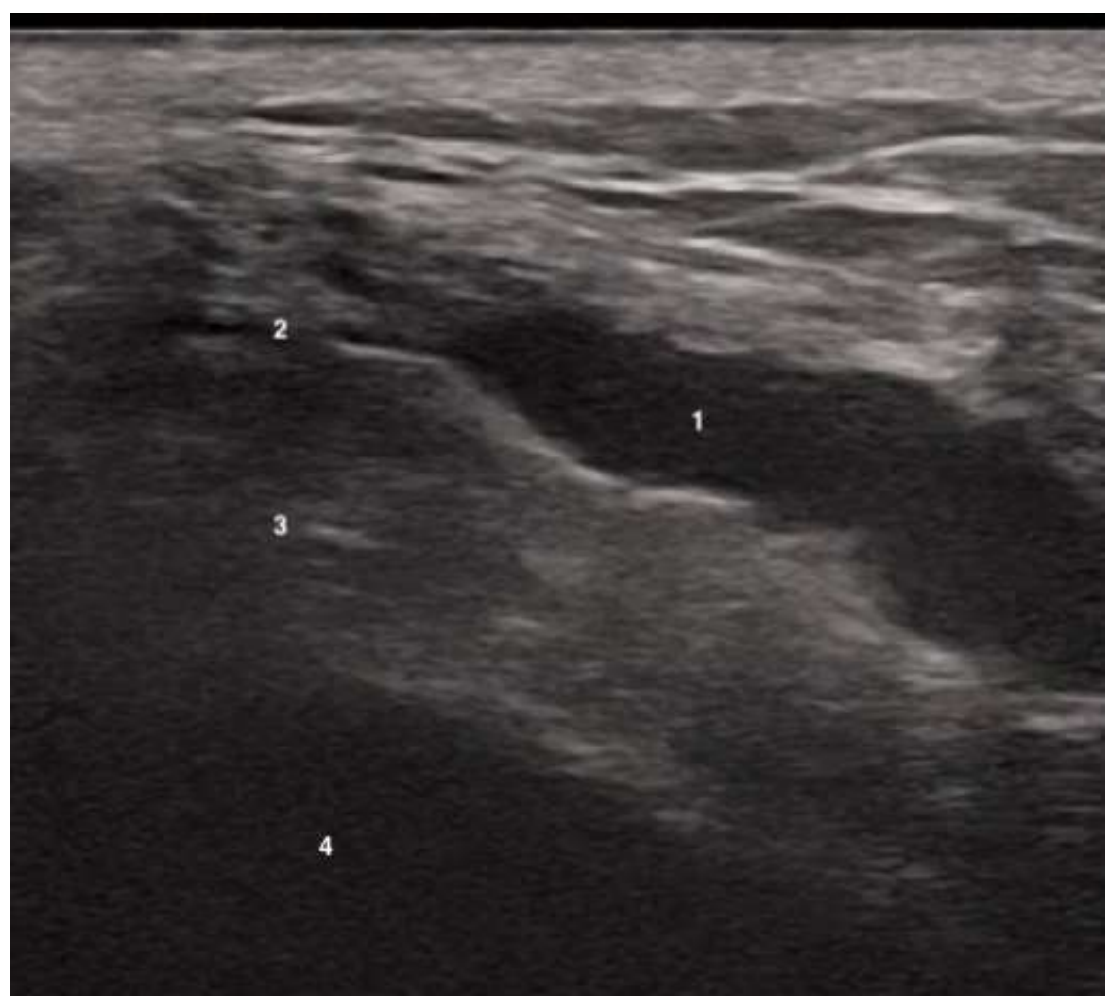

IMAGE 1 Left Knee: 1-LPPB, 2-LPPB Channel,

3-Lateral Supracondylar Fat Pad, 4-LFC

Materials \& Methods:

50 GP and Orthopaedic surgeon referred knee pain patients with transferring issues, received USG SKIF position MPPB-LPPB or channel injections. Two of these injections were performed with the patients remaining in their wheelchairs.

1. Logic E9 machine

2. GE ML6-15-D, 4.5-15.0 Mhz linear transducer.

3. 22 gauge $90 \mathrm{~mm}$ spinal needle

4. 5-10 mg Luer-lock syringes

5. Single-use Optilube packet sterile US gel

6. $4 \%$ Chlorohexidine-Gluconate anti-bacterial antiseptic skin cleanser. 
James Inklebarger et. al. / A Guide to Performing Seated, Foot-On- Floor Position Ultrasound Guided Knee Injections Targeting the Medial \& Lateral Para patellar Bursae \& Their Channels

7. Dressings \& plasters

\section{Patient Positioning}

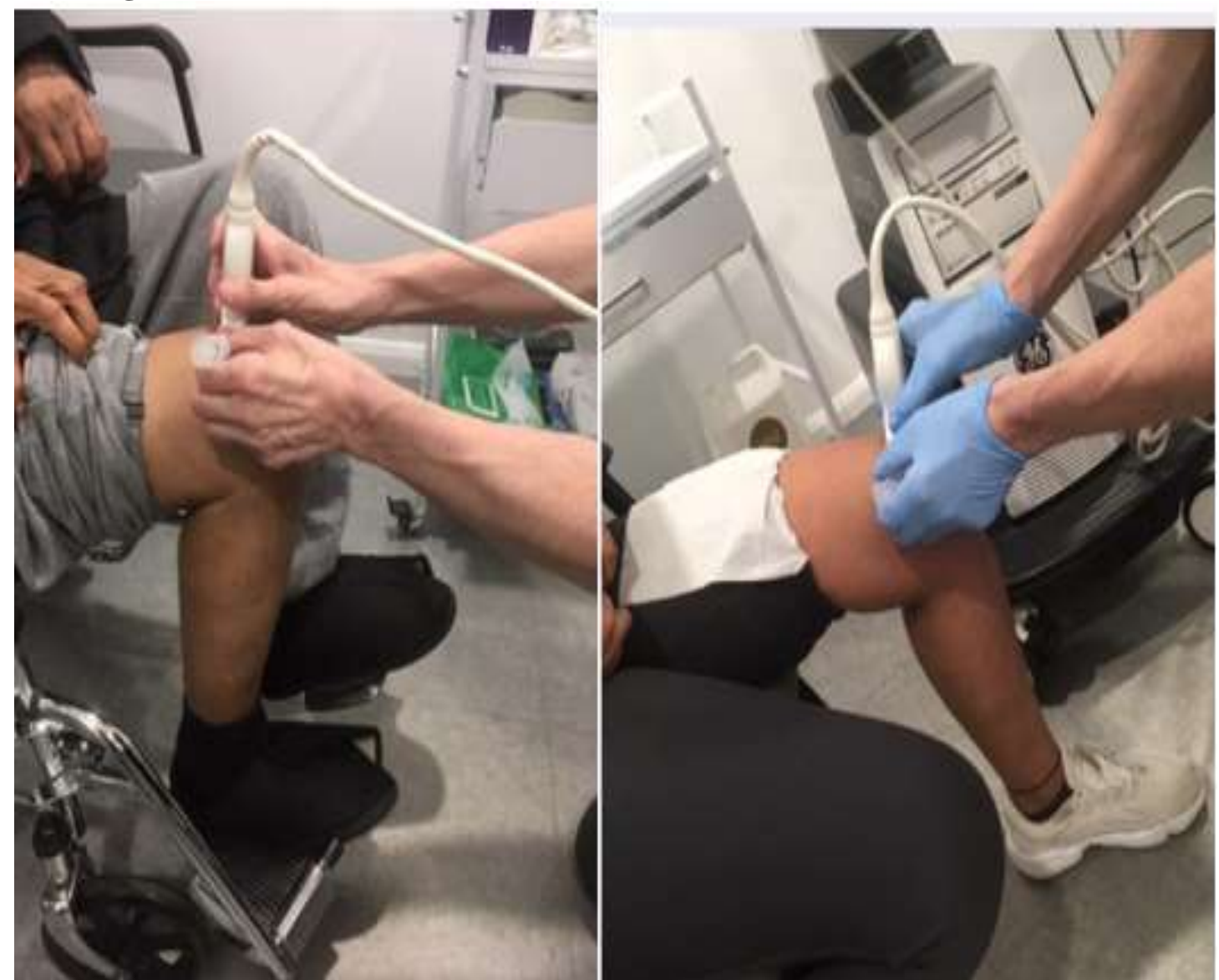

Photos 1 \& 2: USG Injection of the R LPPB in a wheelchair-bound patient and of the L MPPB in a foot-on floor position.

Patients remained seated in a chair with the knee flexed to 90 degrees with the foot planted flat upon the floor or wheelchair pedal. The suprapatellar knee area was first thoroughly cleaned with $4 \%$ Chlorohexidine, [7] and then sterile gel was applied.

The probe was first oriented in the sagittal plan in order to visualize the distal quadriceps tendon, patella, fat pads, and suprapatellar pouch. [Image 2]

Below are longitudinal knee extended-flexed comparative views of the relevant soft tissue and bony anatomical landmarks. Note that in full knee extension, the quadriceps tendon (QT) bows downward and the patella is in a superior position [Image 2], which somewhat obscures the SPB. In 90 degrees knee flexion [Image 3], the patella moves inferiorly lengthening the QT and the SPB is more easily seen. 
James Inklebarger et. al. / A Guide to Performing Seated, Foot-On- Floor Position Ultrasound Guided Knee Injections Targeting the Medial \& Lateral Para patellar Bursae \& Their Channels

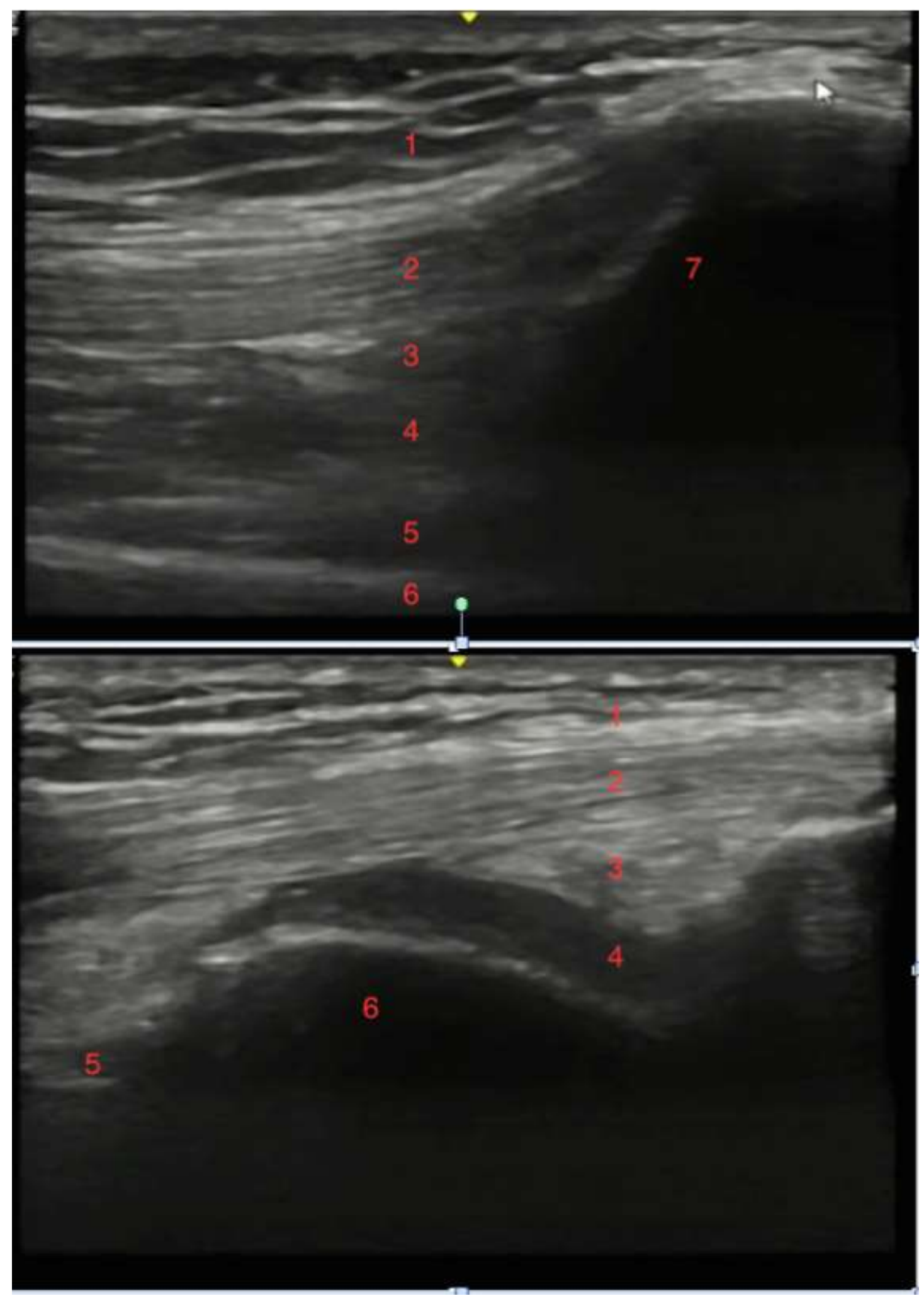

Images 2 \& 3: Comparative Long Axis Knee Extension-Flexion Views: 1-Skin \& subcutaneous tissues, 2Quadraceps tendon, 3-Pre-Quadreceps Fat Pad, 4-Region of the Suprapatellar Bursa, 5-Pre-Femoral Fat Pad, 6-Femur, 7-Patella

After identifying these midline anatomical landmarks in the longitudinal plane, maintain probe position while carefully rotate the probe 90 degrees to obtain a transverse-centralized Supra Patellar view [Image 4] 
James Inklebarger et. al. / A Guide to Performing Seated, Foot-On- Floor Position Ultrasound Guided Knee Injections Targeting the Medial \& Lateral Para patellar Bursae \& Their Channels

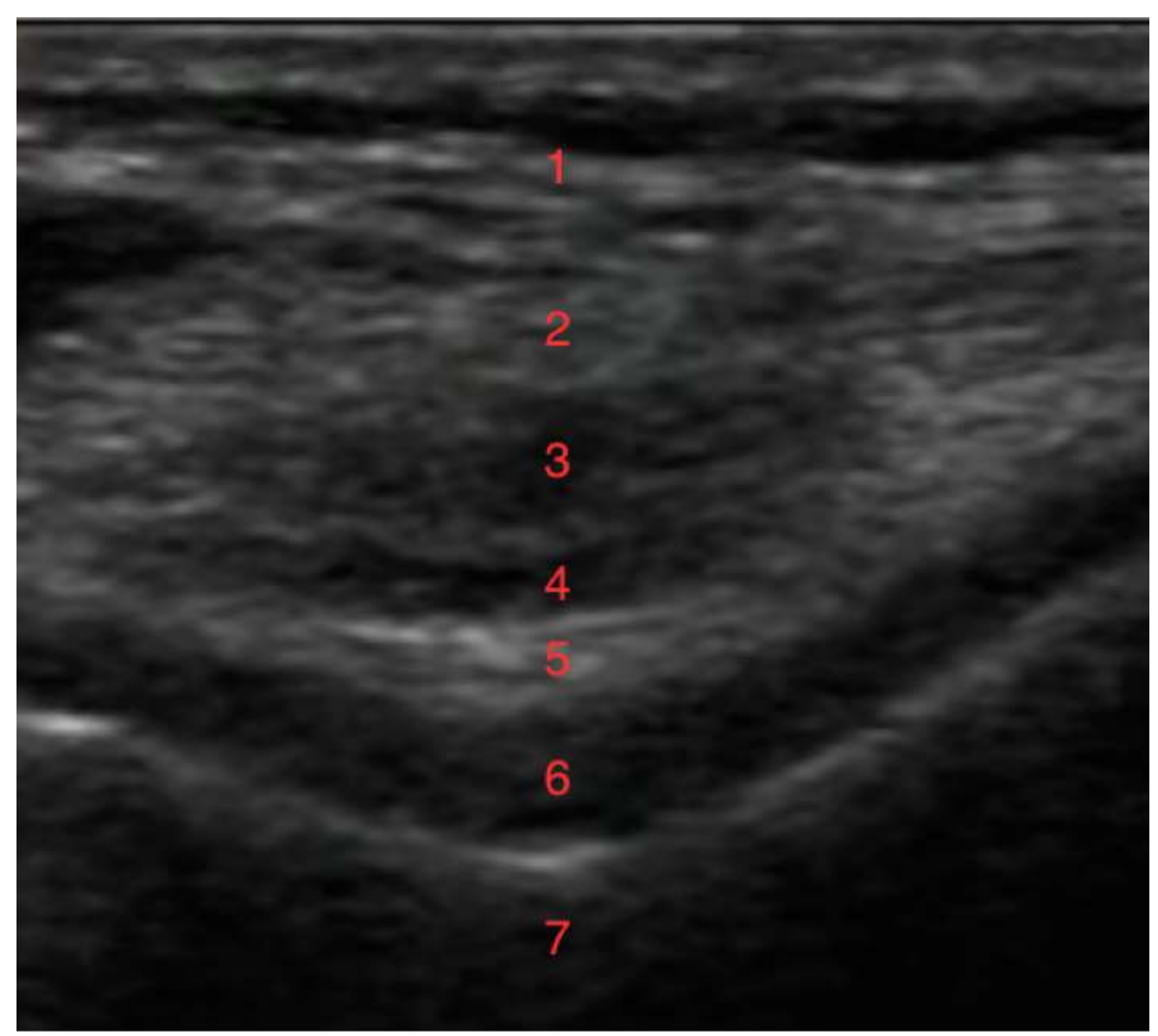

Image 4: Transverse Supra-Patellar view with the knee flexed to 90 degrees: 1-Skin \& Subcutaneous Tissue, 2-Quadraceps Tendon, 3-Pre-Quadriceps Fat Pad, 4-Central Supra-Patellar Recess, 5-Pre-Femoral Fat Pad, 6-Articular Cartilage, 7-Trochlear Groove of the Femur

Suprapatellar fluid was confirmed by fluid compressibility. The probe was then rotated in a transverse plain while maintaining position above the patella to further interrogate the SPB, Lateral-Medial SPB recess, the MPP-LPPB and their channels, with further probe ballottement confirming potential fluid collections. Doppler is then used to map the proximity of vascular structures. USG injections $+/$ - aspiration, were then carried out using a no-touch technique, with a 22 gauge $90 \mathrm{~mm}$ spinal needle using an in-plane approach, and aiming for the most superficial and accessible effusion pocket of the MPPB-LPPB or their connecting channels. Incremental advancement, hydro-dissection with peripheral to central channel expansion confirmed centripedal injectant flow towards the SPB.

\section{Procedure}

1. The entire medial, lateral, and middle aspects of the supra-patellar aspect of the knee was cleaned with Chlorohexidine. Sterile US gel was then placed over the supra-patellar region, ensuring that the site of injection remained gel-free.

2. The MPPB-LPPB \& SPB and inter-bursal channels [Image 1] were identified by compressibility upon probe pressure and their hypo or anaechoic appearance. The knee remained flexed to 90 degrees, with the patient's foot positioned securely on the floor or wheel-chair pedal throughout US examination and guided procedure.

3. The SPB was first identified in knee-flexed long axis, [Image 2 ] and while maintaining probe contact with the skin, rotated orthogonally over the SPB. Brisk, gentle, intermittent on-off transducer 
James Inklebarger et. al. / A Guide to Performing Seated, Foot-On- Floor Position Ultrasound Guided Knee Injections Targeting the Medial \& Lateral Para patellar Bursae \& Their Channels

pressure ballottement will aid identification of the SPB and SPB medial-lateral recesses as compressible fluid pockets. The probe is then moved either medially or laterally in order to look for MPPB-LPPB bursal fluid and their medial-lateral SP recess connecting channels. [Figure 1, Image \& 1]

4. The tri-bursa (SPB-MPPB-LBBP) and medial lateral SPB recess connecting channels are all scanned and dopplered to plan for optimal injection approach. [Figure 1]

5. Finger pressure palpation [black arrow-Image 5 below] may also be used to dynamically locate a less visible SPB recess or MPPB-LPPB between tissue glide planes.

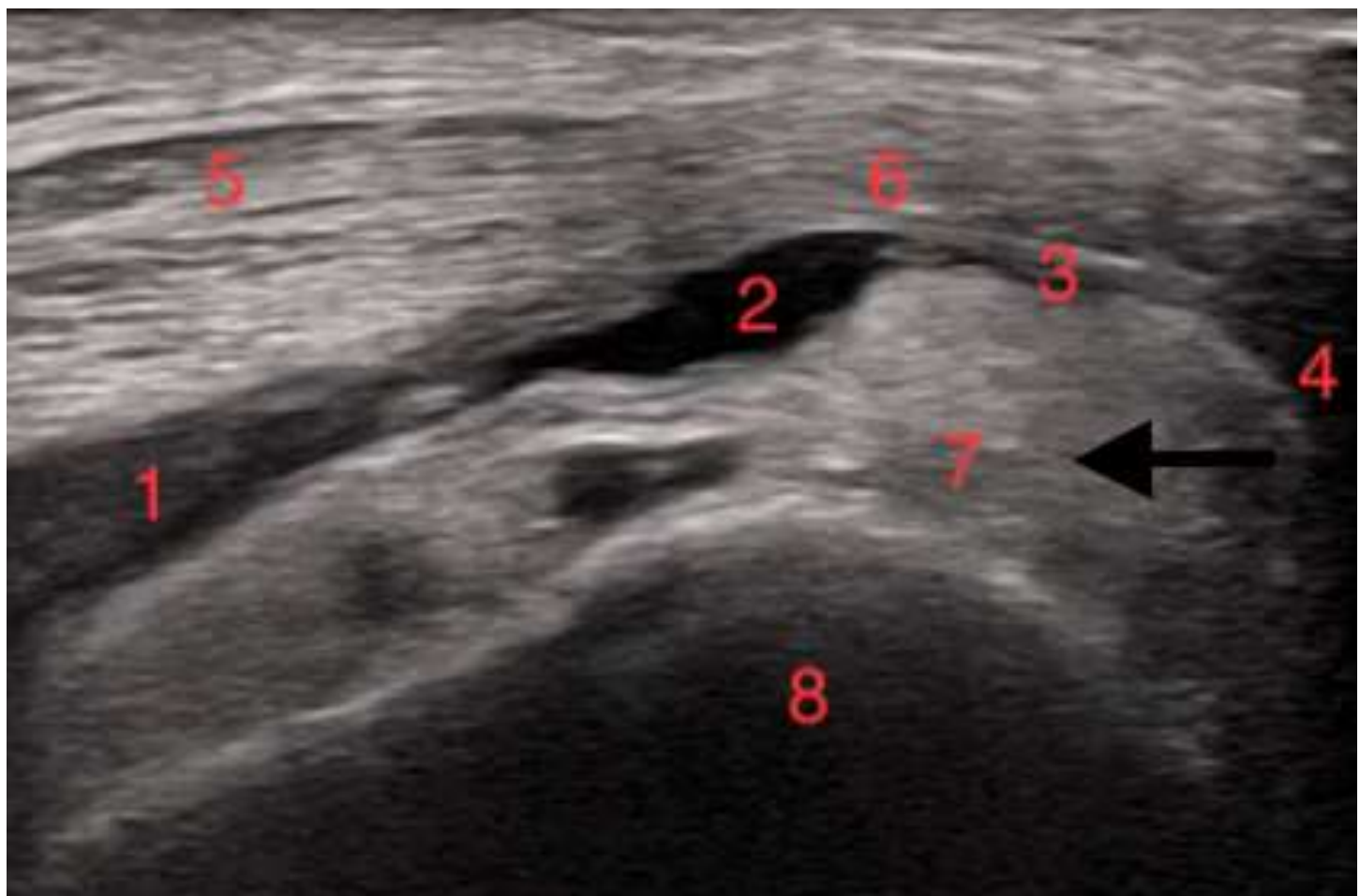

Image 5: Lateral Left Knee: 1-SPB, 2 SPB Lateral Recess, 3-LPPB Channel, 4-LPPB, 5-Lateral aspect of the QT and Vastus Lateralis, 6-Region of the Lateral Pre-Quadriceps Fat Pad (LPFP), 7-Lateral Supracondylar Fat Pad (LSFP), 8-LFC, Arrow: Direction for applying lateral to medial digital pressure against the LSFP to ID its glide motion under the less QT \& LPFP. This manual maneuver may help in locating a not so visible SPB recess \& LPPB Channel, as these spaces are anatomically situated between these glide planes.

\section{Specifics Of MPPB-LPPB Channel \& Bursal Injections}

The MPPB \& LBBP Channels communicate with the medial \& lateral suprapatellar recesses, overlay the medial \& lateral epicondyles, and connect the MPPB-LPPB to the deeper-central SPB. Channel injections may be considered if the MPPB-LPPB are not well visualized for example, due to excessive intra-bursal synovial hypertrophy. When targeting the channels, inject small volumes of fluid while sonographically monitoring for centripedal injectant flow and anaechoic expansion from the channels into the SPB recesses. In this circumstance a needle trajectory to arc the shaft up and over the femoral condyles, may help to prevent potential condylar articular cartilage needle-stick injuries. [Image 6] 
James Inklebarger et. al. / A Guide to Performing Seated, Foot-On- Floor Position Ultrasound Guided Knee Injections Targeting the Medial \& Lateral Para patellar Bursae \& Their Channels

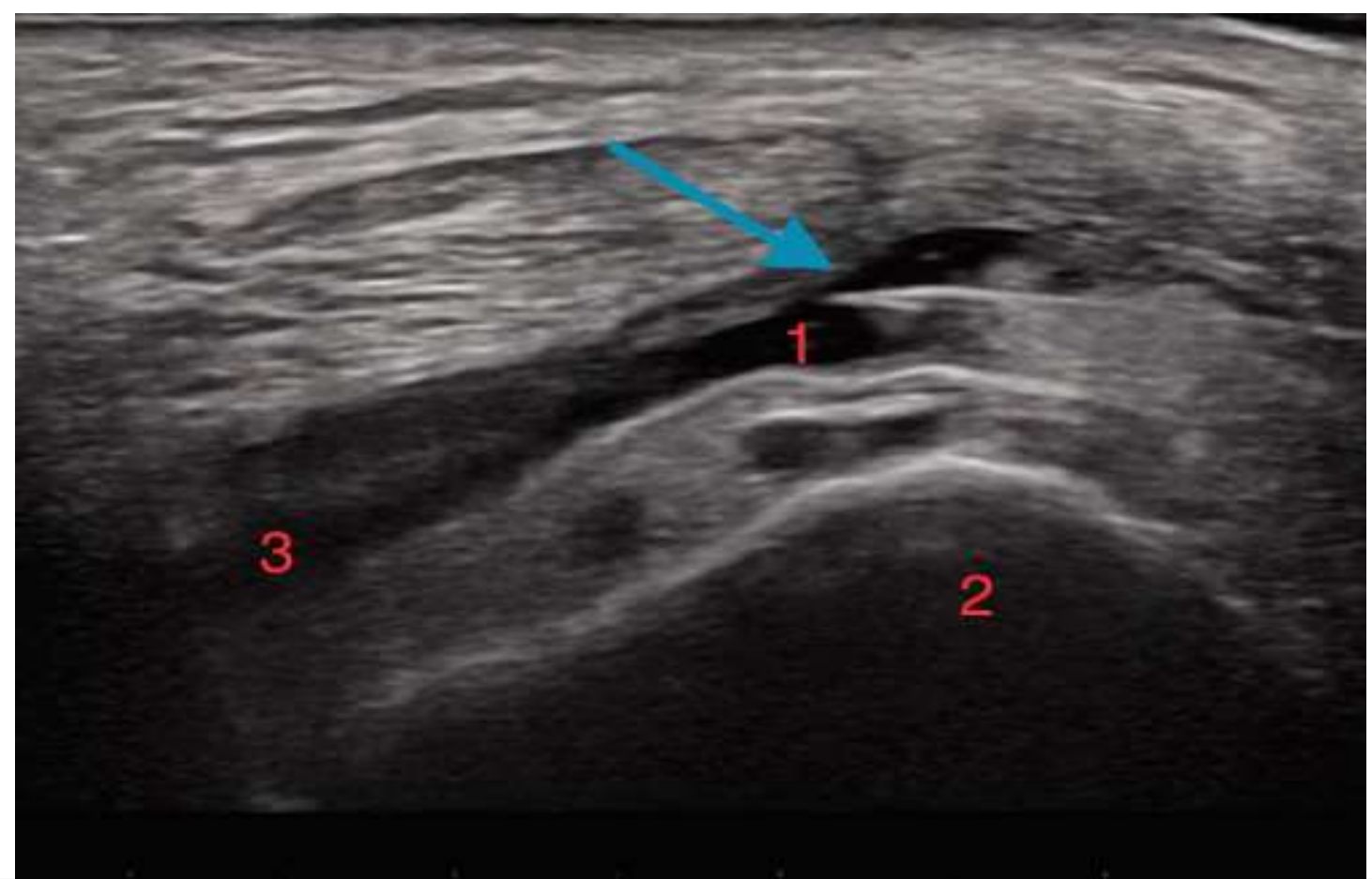

Image 6: Medial Suprapatellar Recess) injection (1), with the needle (blue arrow), angled up \& over the MFC (3) The Role of Ultrasound in Rheumatology Clinical Practice - Lower Extremities, https://youtu.be/EGCgGHNFiU4

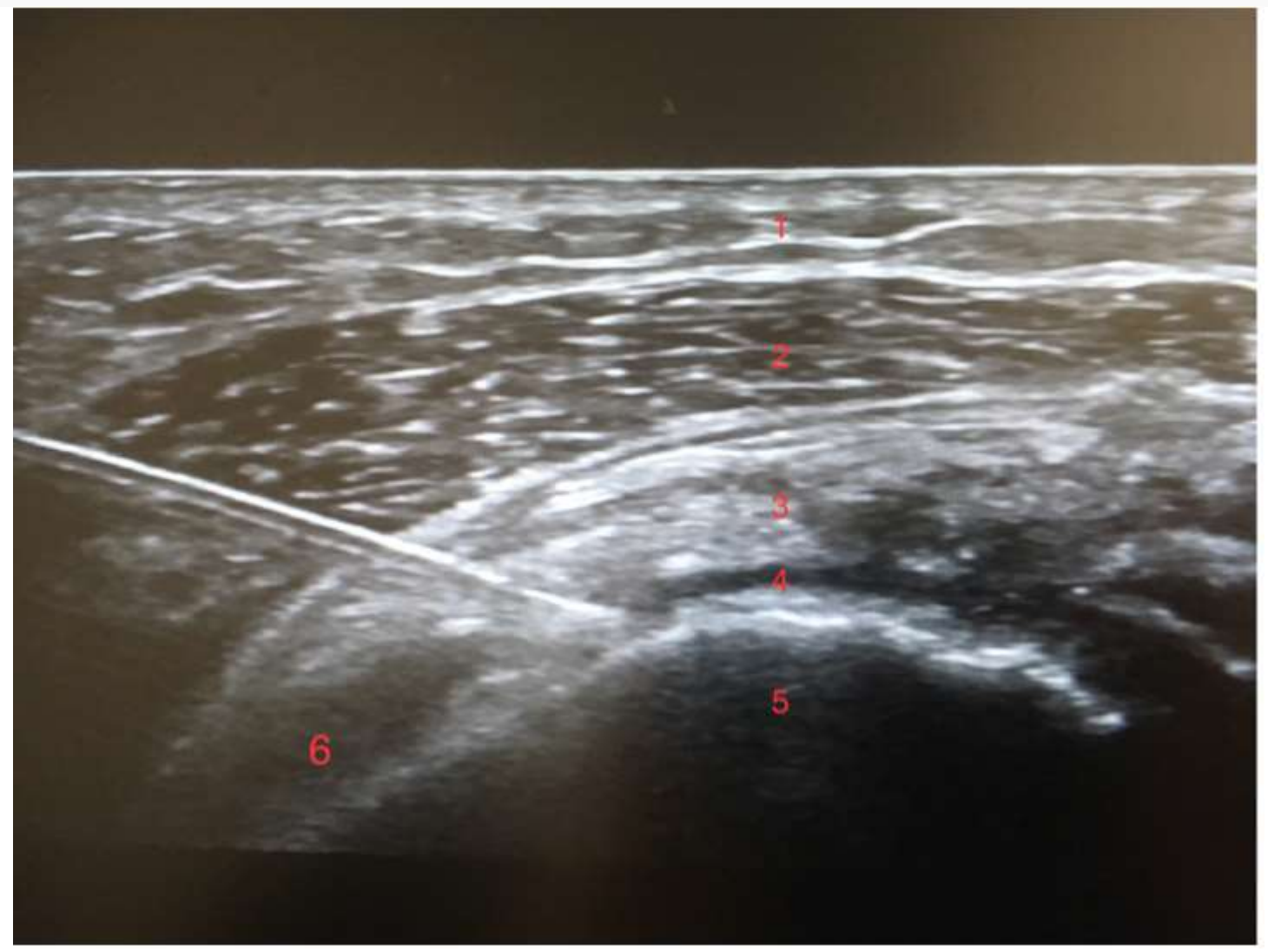

Image 7: Seated MPPB Channel Injection Left Knee: 1-Skin \& subcutaneous tissue, 2-Vastus Medialis, 3Medial Supra-Patellar Fat Pad, : 4-MPPB Channel with injection spread into the deeper SPB, 5-Medial Femoral Condyle (MFC), 6-MBBP. Note: The needle tip does not contact the MFC. 
James Inklebarger et. al. / A Guide to Performing Seated, Foot-On- Floor Position Ultrasound Guided Knee Injections Targeting the Medial \& Lateral Para patellar Bursae \& Their Channels

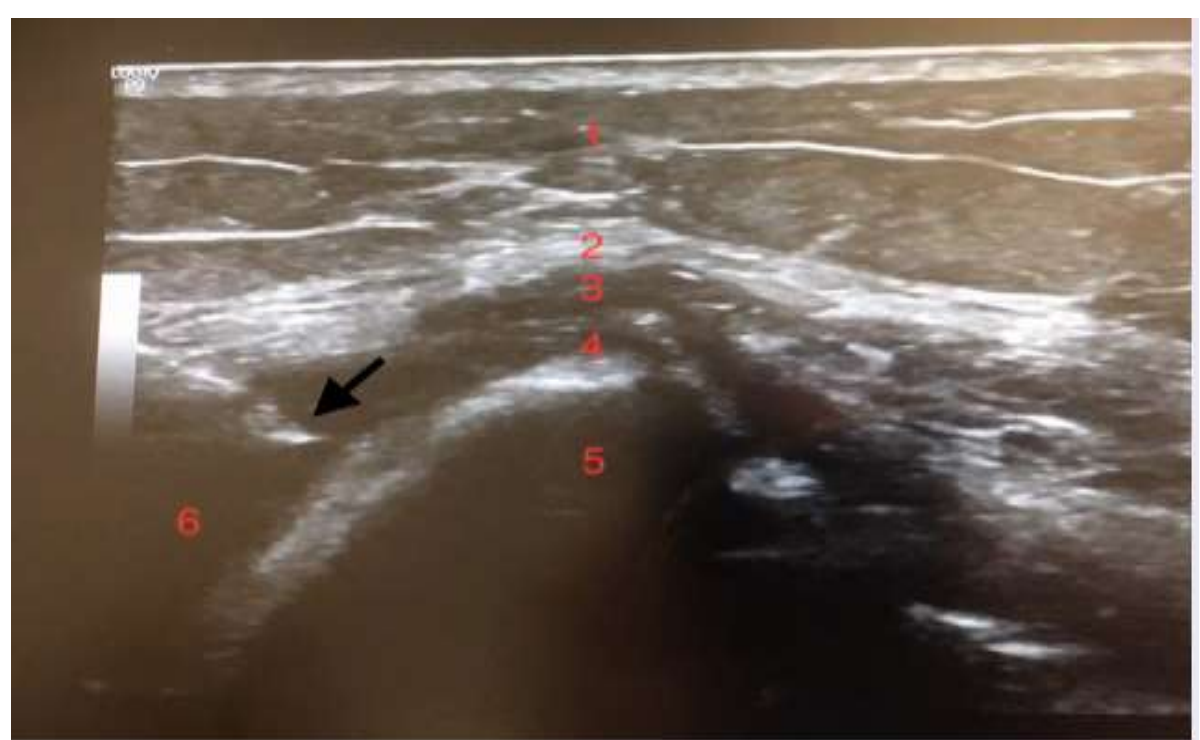

Image 8: Seated Lateral Para-Patellar Bursa (LPPB) Right Knee Hyaluronic acid (Ostenil Plus) injection. This procedure was performed on an 89 year old female: 1- Sub-Cutaneous Skin \& Tissue, 2-Vastus Lateralis, 3-Lateral Supra-Patellar Fat Pad, 4-Communication with injection spread between the LPPR and deeper SPB. 5-Lateral Femoral Condyle, 6-Large, effused anaechoic LPPR. Arrow: Needle Tip

\section{Medial-Lateral SPB Recesses \& MPPB-LPPB \& Channels Identification \& Injection In Hard To See Cases}

Osteoarthritis of the knee is also associated with synovial hypertrophy( $\mathrm{SH})$, and synovitis. [8, 9]

The SPB, SPB medial-lateral recess, channels, and MPPB-LPPB may all be SH obscured. However, motion palpation of the supracondylar fat pads below the medial-lateral pre-quadriceps fat pads may help to indirectly identify the glide planes between these to structures, in which collapsed non-visible medial-lateral SPB recesses \& MPPB-LPPB may be situated. In Image 8 below, a partially obscured lateral

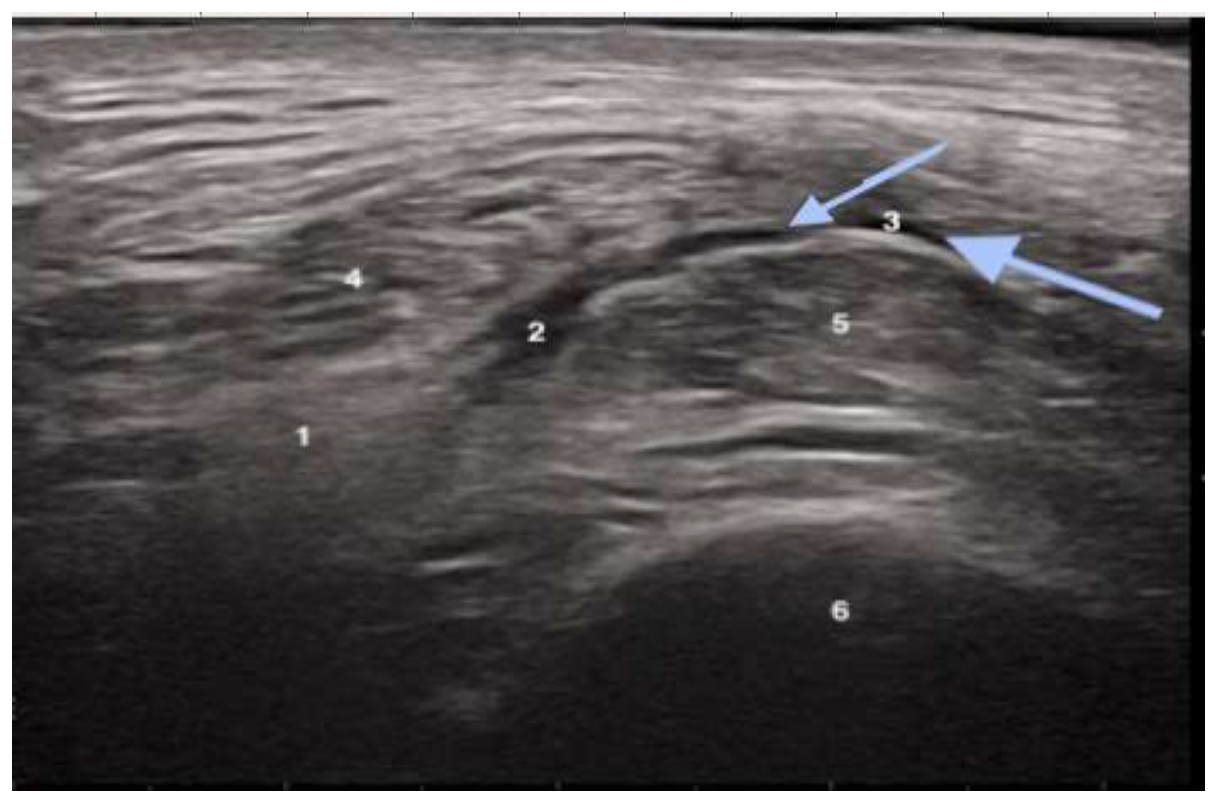

Image 9: Left Lateral Knee: 1-Obscured SPB, 2-Partially obscured Lateral SPB recess (LSPBR) injection target, 3-Obscured LPPB Channel, Black Arrow-Arc of needle travel threading into the LSPBR, 4-Obscured QT \& Lateral Pre-Quadriceps Fat Pad (PQFP), 5-Lateral Supracondylar Fat Pad, 6-LFC, Small Blue Arrow: Needle vector option for injection of the LPPBR channel. Larger arrow: Vector option for injecting into the LPPB channel. 
James Inklebarger et. al. / A Guide to Performing Seated, Foot-On- Floor Position Ultrasound Guided Knee Injections Targeting the Medial \& Lateral Para patellar Bursae \& Their Channels

These spongy \& boggy tissue growths may become florid and present as waving fronds of tissue [Image 9 below], or they may partially or completely obscure knee the tri-bursae and channels. Additionally intrabursal overgrowth may present obstruction to aspiration- injection (lemon seed in straw) and necessitate needle tip repositioning or steerage around achieve SH for procedure success.

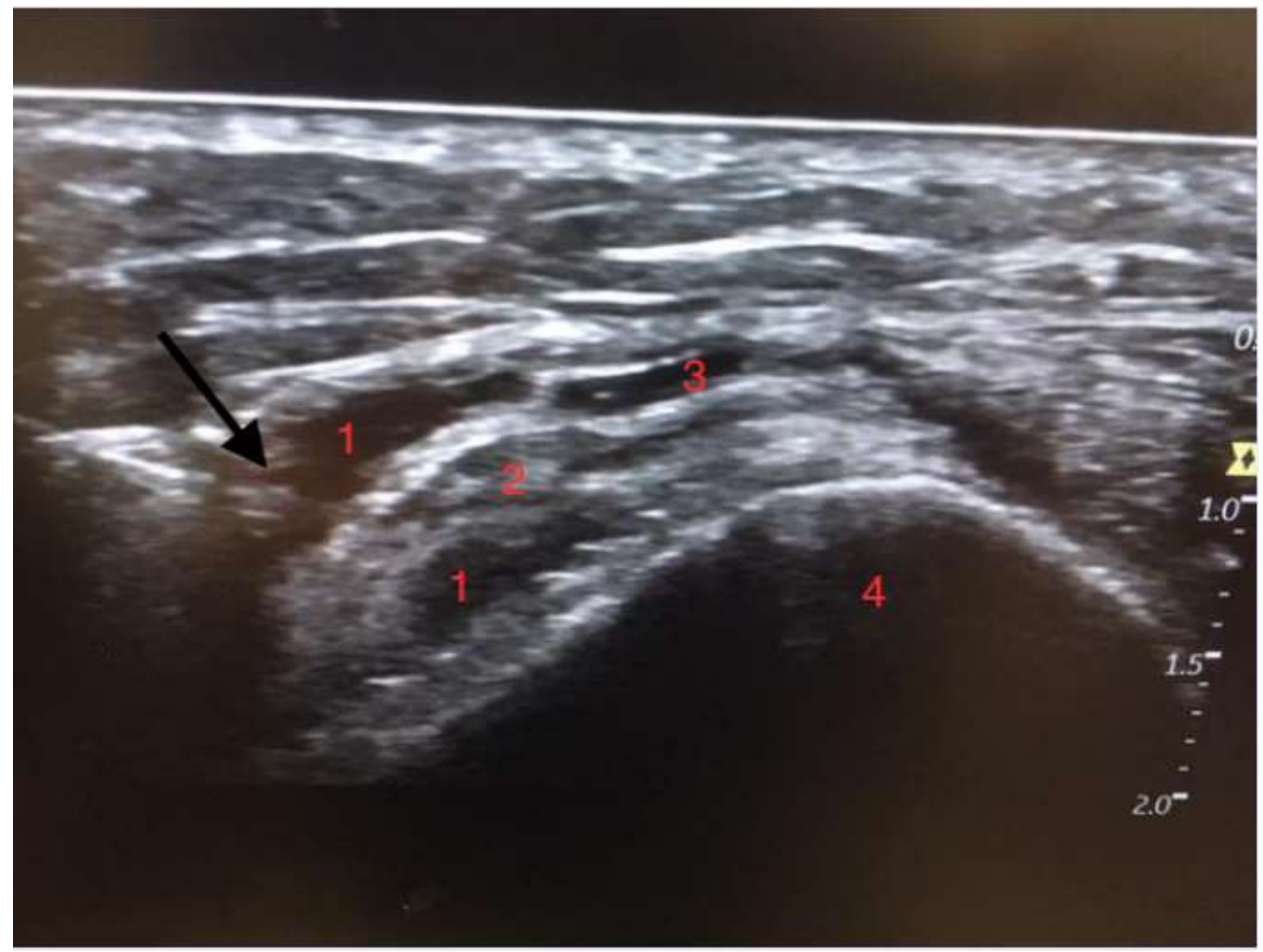

Image 10 R Knee: (1) LPPB with florid intra-bursal SH (2), Intra-Bursal channel expansion confirming centripedal SPB injectant flow (3), 4-LFC, Black Arrow: Needle tip

\section{Some Take Home Skif Technique Points}

1. Pre-scan and plan your aspiration-injection target prior to procedure.

2. Ballotable-compressible hypo-anechoic fluid is a sign a bursa is being visualized. Avoid probe overpressure which may obscure the bursa.

3. Digital inward pressure in on the medial or lateral supracondylar fat pad (SCFP) may indirectly detect hard to locate the anatomical position of the medial-lateral SPB recesses or bursal channels in the glide plane between the SCFP and the PQFP.

4. If targeting the MPPB-LPPB inter-bursal channels, 22-gauge needle flexibility facilitates re-directs, while its diameter prevents inadvertent cartilage penetration as may occur with a smaller diameter needle, while also providing for adequate aspiration-injection flow.

5. Easy injection resistance is an indicator if proper needle position within the bursa. If excessive resistance to aspiration-injection flow is encountered, reposition the needle tip.

6. In $\sim 15$ percent of cases, the SBP and ergo the MPPB-LPPB and inter-bursal channels do not communicate with the knee joint. This may partially explain some procedure failures and if suspected, and alternative knee injection site (infra-patellar) may be considered.

7. If during procedure the needle tip is not visualized well, inject a small amount of fluid to detect fluid expansion. Maneuvering and flushing of the needle may also be required to clear SH debris blockages. 
8. Though the MPPB-LPPB locations are devoid of articular cartilage, they do overlay a nocicepter rich periosteum. Avoidance of inadvertent needle tip periosteal contact will make the procedure more comfortable.

\section{Discussion}

The suprapatellar bursa (SBP) is known interchangeably as the suprapateller recess or suprapatellar bursa[10]. Failure of regression of the transverse septum formed in embryonic life between the suprapatellar plica and the knee joint fluid leads to the formation of the suprapatellar bursa (SPB). [11]

It is an extension of the intrarticular synovial cavity, as with all bursae, its purpose is intended to reduce friction between moving structures facilitating flexion-extension movement of the quadriceps tendon over the femur.

Cross-sectional SPB effusion ultrasound measurements of suprapatellar effusion have noted a qualitative association with symptoms of radiographically diagnosed knee OA. [12]

Sonographically, the SPB appears as a well-defined hypoechoic band, cranial to patella, anterior to the distal femoral shaft and posterior to quadriceps tendon, between the prefemoral and SPB fat pads. US-guided SPB knee injection exhibit a significantly greater accuracy (48 of 50 knees, 96.0\%) than blind injection (41 of 49 knees, 83.7\%). Intra-articular injections through the suprapatellar bursa under US guidance increased the accuracy of knee joint injections. [13], and also improves accuracy in the obese and in conditions such as lymphedema. [14]

Randomized controlled trials of anatomical knee landmark injection comparison studies, have also noted less pain, better outcomes, patient satisfaction scores, and improved accuracy knee $(96.7 \%$ versus $81.0 \%, \mathrm{P}$ $<0.001$ ), with US guidance [14. Fang et al 2018], with the suprapatellar bursa being the most common target. [15]

Martino and colleagues noted that voluntary quadriceps concentric contraction (knee extension) significantly increases visualization of the SPB from $16 \%$ to $66 \%$.

[16]

However, USG SBP aspiration-injection, is typically performed on a plinth, with the patient in a SemiFowler 30 degree knee-flexed position.

This 30-degree knee-flexed position correlates to a recess fluid visibility study, which compared varying degrees of knee flexion $(0,15,30,60,90$ degrees, and concluded that the suprapatellar and medial-lateral parapatellar recess fluid is optimally visualized with the knee in 30 degrees flexion.[4]

However, a plinth-seated USG SPB injection technique with the knee flexed and dangling (90 degrees flexion) has also been proposed by [17]

Furthermore a surface EMG bench-foot push-off study, noted that isometric quadriceps contraction was overall greater at 90 degrees of knee flexion. [5. Morrish GM et al 2003] Furthermore, Wada et al also devised a supine, plinth-positioned, knee flexed, voluntary isometric quadriceps contraction supralateral knee injection approach, which also demonstrated enhanced US resolution of the SPB, [18]

In summary, 'following the fluid' rather than rote routine optimizes safety and success. Weigh and balance tri-bursal or channel injection selection choices on factors of safety, optimal image generation, ease of needle steerage around obstructions (intra-bursa synovial hypertrophy, osteophytes), and plan for the least distance of needle travel to achieve target acquisition.

\section{Pros \& Cons of Skip Foot-On Floor MPPB-LPPB \& Channels Injection Aspiration Summary:}

Whereas the SPB, its recesses, and the MPPB-LPPB channels overlay cartilage, the areas underlying the MPPB-LBBP areas are articular cartilage devoid. This is an important consideration, as even the most benign superficial injuries to articular cartilage including temporary deformation may result in chondrocyte death. [19]. Due to the avascular, aneural, and aliphatic nature of cartilage it possesses a poor capacity for healing. Some studies have hypothesized that inadvertent needle stick injury during cartilage suturing, creat channel-like defects, associated with damage to the adjacent tissue. Needle-stick lesions may not only fail to heal but may enlarge with time and joint usage. [20] 
Post arthroscopic chondrolysis has been reported most often in the gleno-humeral joint but has also been documented in the knee joint too. [21, 22]

Articular cartilage overlaying the medial \& lateral femoral condyles and is subject to extremes of contact pressures at end of range knee flexion-extension. [23]

Therefore, when targeting MPPB-LPPB channels overlaying the condyles and as it is with SPB injections, care must be taken to avoid inadvertent underlying cartilage needle stick injury.

Studies have described that optimal intracondylar hyaline cartilage resolution is achieved with the probe in transverse suprapatellar plane with the knee flexed to 90 degrees. [24] If the structures with underlying cartilage are to be targeting for injection-aspiration, it therefore follows that the SKIF position approximates of 90 degrees of knee flexion, may also optimally distinguish cartilage from closely approximated bursal structures, thereby prevented procedural cartilage needle sticks.

Though the SPB is a common target for knee aspiration-injection there are other pitfalls to successful tribursal, recess \& channel injection procedures. Studies have noted that in $\sim 15$ percent of the population the SBP and ergo the MPPB-LPPB \& channels also may not communicate with the knee joint proper, defeating intra-articular injectant spread. There are also 4 types of remnant embryonic septum variants which influence the synovial fluid circulation between these bursae and intra-articular joint. [25, 26]

These bursal morphological variants may offer some insight into potential anatomical reasons for suboptimal procedure outcomes. If procedure failure is suspected for these anatomical reasons another knee injection (infra-patellar) may be considered. [27]

\section{Conclusion}

The MPPB-LPPB structures are largely under-reported in the literature, while the MPPB-LPPB and it appears that tri-bursal channel anatomy has not previously described or defined. As it is with the SPB, the variable visibility of these tri-bursal structures and their communicating channels may be significantly enhanced by quadriceps muscle contraction. [28, 15] A Seated, foot planted and the knee flexed to 90 degrees (SKIF) procedure may therefore generate quadriceps contraction-enhanced tri-bursal structure resolution via foot push off counter-force, thereby brightening injection targets.

Preferential targeting of the MPPB-LPPB, may be also offer an anatomically safer technique, by avoidance of articular cartilage needle sticks. Their relatively superficial paracondylar anatomy may also offer a relatively more efficient knee injection-aspiration alternative, in cases of poor SBP visualization challenged by bursal collapse, a thickened SP plica, scarring, or obscured by florid intra-bursal proliferative synovitis tissue hypertrophy. USG SKIF position [Photos $1 \& 2$ ] may also offer an alternative choice for those in pain, wheelchair-bound, or who may otherwise experience difficulties with plinth transfer.

As some of the structures described and imaged in this paper (MPPB-LPPB) have been sparsely reported or appear not to have yet been reported at all, (MPPB-LPPB channels), further be will be required to delineate their anatomy, and the reproducibility and efficacy of this novel approach to USG knee injection.

\section{Acknowledgements:}

Special thanks to Payal Khambhaita for administrative support \& Ken Faulkner for graphic art design.

\section{$\underline{\text { References }}$}

1. Koh WL, Kwek JW, Quek ST, Peh WC. Clinics in diagnostic imaging (77). Pes anserine bursitis. Singapore Med J. 2002;43:485-91. [PubMed] [Google Scholar]

2. Zidorn T, Tillmann B. Morphological variants of the suprapatellar bursa. Ann Anat. 1992 Aug;174(4):287-91. doi: 10.1016/s0940-9602(11)80284-0. PMID: 1416055.

3. Gilcrease-Garcia, B., Hacking, C. Suprapatellar bursa. Reference article, Radiopaedia.org. (accessed on 23 Dec 2021) https://doi.org/10.53347/rID-62254

4. Mandl P, Brossard M, Aegerter P, Backhaus M, Bruyn GA, Chary-Valckenaere I, Iagnocco A, Filippucci E, Freeston J, Gandjbakhch F, Jousse-Joulin S, Möller I, Naredo E, Schmidt WA, Szkudlarek M, Terslev L, Wakefield RJ, Zayat A, D'Agostino MA, Balint PV. Ultrasound evaluation 
of fluid in knee recesses at varying degrees of flexion. Arthritis Care Res (Hoboken). 2012 May;64(5):773-9. doi: 10.1002/acr.21598. PMID: 22232128.

5. Morrish GM, Woledge RC, Haddad FS. Activity in three parts of the quadriceps recorded isometrically at two different knee angles and during a functional exercise. Electromyogr Clin Neurophysiol. 2003 Jul-Aug;43(5):259-65. PMID: 12964252.

6. Marco A. Cimmino, Dario Camellino, Francesco Paparo, Silvia Morbelli, Michela Massollo, Maurizio Cutolo, Gianmario Sambuceti, High frequency of capsular knee involvement in polymyalgia rheumatica/giant cell arteritis patients studied by positron emission tomography, Rheumatology, Volume 52, Issue 10, October 2013, Pages 1865-72, https://doi.org/10.1093/rheumatology/ket229

7. Stinner DJ, Krueger CA, Masini BD, Wenke JC. Time-dependent effect of chlorhexidine surgical prep. J Hosp Infect. 2011 Dec;79(4):313-6. doi: 10.1016/j.jhin.2011.08.016. Epub 2011 Oct 15. PMID: 22000737.

8. Ene R, Sinescu RD, Ene P, Cîrstoiu MM, Cîrstoiu FC. Synovial inflammation in patients with different stages of knee osteoarthritis. Rom J Morphol Embryol. 2015;56(1):169-73. PMID: 25826502.

9. Mathiessen A, Conaghan PG. Synovitis in osteoarthritis: current understanding with therapeutic implications. Arthritis Res Ther. 2017;19(1):18. Published 2017 Feb 2. doi:10.1186/s13075-0171229-9

10. Hacking C, Gilcrease-Garcia, Surpatellar Bursa, Radiopaedia, 2021, Radiopaedica.org/articles/suprapatellar-bursa?lang=gb

11. Dorsey ML, Liu PT, Leslie KO, Beauchamp CP. Painful suprapatellar swelling: diagnosis and discussion. Skeletal Radiol. 2008 Oct;37(10):937-8, 951-2. doi: 10.1007/s00256-008-0547-z. PMID: 18651141.

12. Chiba, D., Tsuda, E., Maeda, S. et al. Evaluation of a quantitative measurement of suprapatellar effusion by ultrasonography and its association with symptoms of radiographic knee osteoarthritis: a cross-sectional observational study. Arthritis Res Ther 18, 181 (2016). https://doi.org/10.1186/s13075-016-1078-y

13. Bum Park Y, Ah Choi W, Kim YK, Chul Lee S, Hae Lee J. Accuracy of blind versus ultrasoundguided suprapatellar bursal injection. J Clin Ultrasound. 2012 Jan; 40(1):20-5. doi: 10.1002/jcu.20890. Epub 2011 Oct 28. PMID: 22033897.

14. Fang WH, Xiao MS, Chen T Thomas MDC, Vangsnessa T Jr, Ultrasound-Guided Knee Injections Are More Accurate Than Blind Injections: A Systemic Review of Randomized Controlled Trails, Arthroscopy, Sports Medicine, and Rehabilitation, 2021, 3(4);1177-87

15. Peng PW, Shankar H: Ultrasound-guided interventional procedures in pain medicine: A review of anatomy, sonoanatomy, and pro- cedures: Part V. Knee joint. Reg Anesth Pain Med 2014; 39:36880 iscepi

16. Martino F, Angelelli G, Ettorre GC, Macarini L, Patella V, Moretti B, Pesce V, Nardella M. Aspetto normale della borsa sovrarotulea nell'ecografia del ginocchio [The normal aspect of the suprapatellar bursa in echography of the knee]. Radiol Med. 1992 Jan-Feb;83(1-2):43-8. Italian. PMID: 1557543.

17. Sadeghi N, Kumar A, Kim J, Dooley J, Images in Anesthesiology: Ultrasound-guided Intraarticular Knee Injection, Anesthesiology 2017; 126-565.

18. Wada M, Fujii T, Inagaki Y, Nagano T, Tanaka Y. Isometric Contraction of the Quadriceps Improves the Accuracy of Intra-Articular Injections into the Knee Joint via the Superolateral Approach. JB JS Open Access. 2018;3(4):e0003. Published 2018 Oct 23. doi:10.2106/JBJS.OA.18.0000

19. Harris JD, Brand JC, Rossi MJ, Leland JM, Lubowitz JH. Iatrogenic Arthroscopic Cartilage Injury: Arthroscrapes Result From Iatrogenesis Imperfecta. Arthroscopy. 2020 Aug;36(8):2041-2042. doi: 10.1016/j.arthro.2020.06.003. PMID: 32747053.

20. Hunziker EB, Stähli A. Surgical suturing of articular cartilage induces osteoarthritis-like changes. Osteoarthritis Cartilage. 2008;16(9):1067-1073. doi:10.1016/j.joca.2008.01.009 
21. Fester E., Noyes M.D.F. 2009. Postoperative chondrolysis of the knee: 3 case reports and a review of the literature. [PubMed] [Google Scholar]

22. Slabaugh M.A., Friel N.A., Cole B.J. Rapid chondrolysis of the knee after anterior cruciate ligament reconstruction: a case report. J Bone Joint Surg Am Vol. 2010 Jan;92(1):186189. [PubMed] [Google Scholar]

23. Shah RF, Martinez AM, Pedoia V, Majumdar S, Vail TP, Bini SA. Variation in the Thickness of Knee Cartilage. The Use of a Novel Machine Learning Algorithm for Cartilage Segmentation of Magnetic Resonance Images. J Arthroplasty. 2019;34(10):2210-2215. doi:10.1016/j.arth.2019.07.022

24. Kauppinen K, Casula V, Zbýň Š, Blanco Sequeiros R, Saarakkala SS, Nevalainen MT. Ultrasonographic Assessment of the Normal Femoral Articular Cartilage of the Knee Joint: Comparison with 3D MRI. ScientificWorldJournal. 2021;2021:9978819. Published 2021 Aug 18. doi:10.1155/2021/9978819

25. Steinbach LS, Stevens KJ. Imaging of cysts and bursae about the knee. (2013) Radiologic clinics of North America. 51 (3): 433-54. doi:10.1016/j.rcl.2012.10.005 - Pubmed

26. Zidorn T, Schäfer H. Morphologic variants of the proximal knee-joint cavity. An anatomical and radiological study. (1992) Surgical and radiologic anatomy : SRA. 14 (2): 141-6. Pubmed

27. Maricar N, Parkes MJ, Callaghan MJ, Felson DT, O'Neill TW. Where and how to inject the knee--a systematic review. Semin Arthritis Rheum. 2013 Oct; 43(2):195-203. doi: 10.1016/j.semarthrit.2013.04.010. Erratum in: Semin Arthritis Rheum. 2015 Apr;44(5):e18. Erratum in: Semin Arthritis Rheum. 2015

28. Sibbitt WL Jr, Kettwich LG, Band PA, Chavez-Chiang NR, DeLea SL, Haseler LJ, Bankhurst AD. Does ultrasound guidance improve the outcomes of arthrocentesis and corticosteroid injection of the knee? Scand J Rheumatol. 2012 Feb;41(1):66-72. 